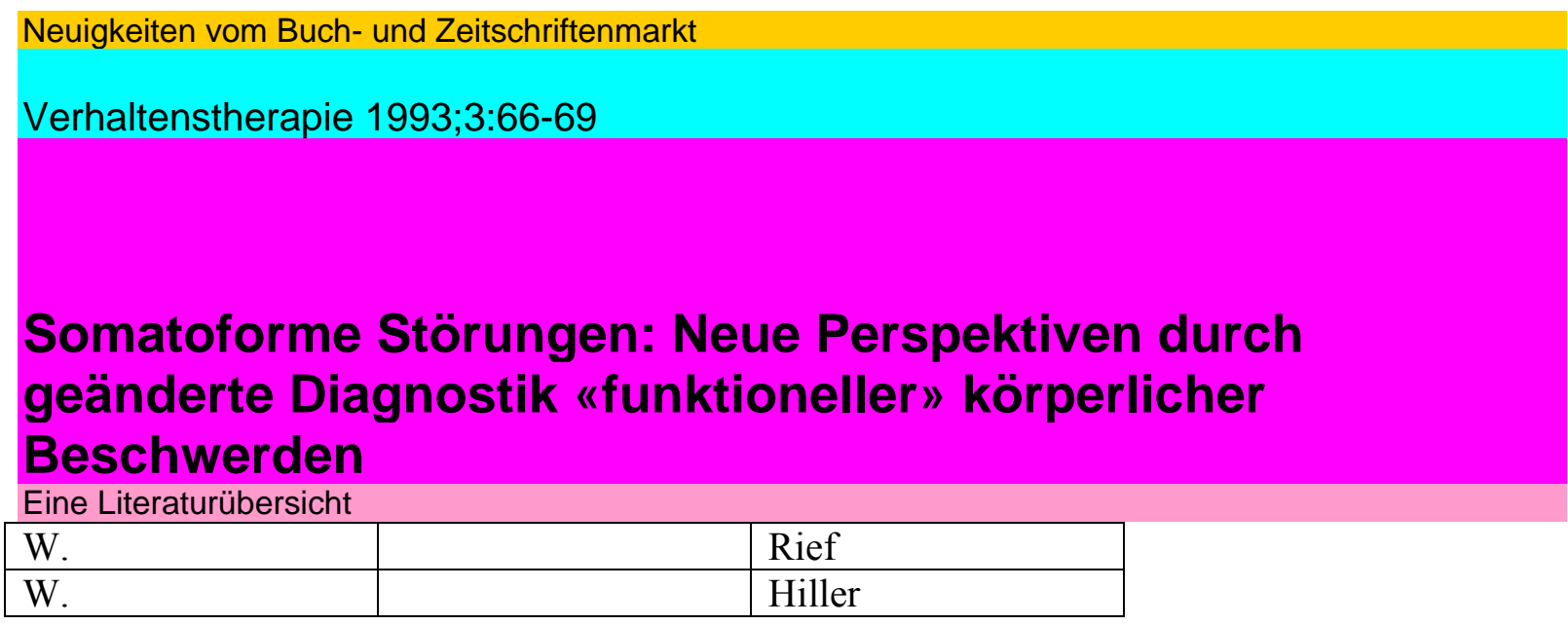

Prien/Chiemsee

Anschrift der Autoren: Winfried Rief, Wolfgang Hiller, Klinik Roseneck, Am Roseneck 6, W-8210 Prien/Chiemsee Einleitung

Hauptmerkmal somatoformer Störungen sind körperliche Beschwerden, die für den Betroffenen mit erheblichen Leiden verbunden sind und trotz zahlreicher körperlicher Untersuchungen nicht auf eine somatische Erkrankung zurückzuführen sind. Somatoforme Störungen werden weiterhin abgegrenzt von Angststörungen, vor allem Panik-störungen, bei denen zwar auch körperliche Beschwerden im Vordergrund stehen können, diese jedoch in der Regel auf kurze Attacken beschränkt sind. Auch Depressionen gehen oftmals mit körperlichen Beschwerden, wie Müdigkeit, Appetitverlust oder Sexualstörungen, einher, die jedoch typi-scherweise Bestandteil eines depressiven Syndroms sind und für sich genommen noch nicht die Diagnose einer somatofor-men Störung rechtfertigen.

Die historischen Wurzeln der somatoformen Störungen lie-gen zum einen im Konzept der Hysterie, zum anderen im Konzept der Hypochondrie. Die mit diesen Konzepten ver-bundenen Diagnosen zeigten sich in der Vergangenheit als wenig reliabel und valid. Mit dem amerikanischen Diagnose-system DSM-III wurde 1980 der Begriff der somatoformen Störungen eingeführt, der sich schwerpunktmäßig auf de-skriptive Merkmale stützt. Über diese Neufassung liegen bis-lang nur wenig Informationen und Forschungsbefunde vor. In den vergangenen 13 Jahren wurde vor allem im diagnosti-schen Bereich geforscht, so daß nun eine Verbreitung des neu erworbenen Wissens über diese Krankheitsformen ansteht. Die nachfolgende kommentierte Publikationsliste soil hier-bei eine kleine Hilfestellung leisten. Die Auswahl kann und soil nicht vollständig sein, sondern den Einstieg in die Thematik erleichtern.

Buchpublikationen

Kellner, R.: Somatization and Hypochondriasis. New York, Praeger, 1986

Dieses Buch gehört sicherlich zu den umfassendsten und fun-diertesten Arbeiten, die in den letzten Jahren zum Bereich der funktionellen Störungen vorgelegt wurden. Der Autor, Professor an der University of New Mexico in Albuquerque, hat sich seit den 60er Jahren in einer Vielzahl von Studien mit dem klinischen Erscheinungsbild, der Diagnostik und der Therapie von Somatisierung und Hypochondrie befaßt. Ins-besondere beruhen seine Arbeiten auf Untersuchungen in

Allgemeinarztpraxen, in denen in den westlichen Industrie-nationen die meisten Patienten mit (häufig lange unerkann-ten) somatoformen Störungen zu finden sein dürften. Das Buch stellt eine gelungene Verbindung zwischen den Störungsbildern der Somatisierung und der 
Hypochondrie her, da Gemeinsamkeiten, aber auch Unterschiede herausge-arbeitet und durch eine übersichtlich aufgearbeitete Durch-sicht der Literatur belegt werden. Das Buch ist übersichtlich gegliedert in Kapitel zur historischen Entwicklung der Störungskonzepte, zu prädisponierenden und aufrechter-haltenden Bedingungen, aber auch zur Diagnostik und zu pharmakologischen und psychotherapeutischen Ansätzen. Insbesondere im diagnostischen Teil findet sich eine gute $\mathrm{Zu}$-sammenstellung von Selbst- und Fremdbeurteilungsansätzen, wobei

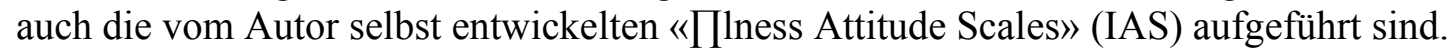
Insgesamt gibt dieses Werk einen breiten und gut verständlichen Überblick, der auch einen Einstieg in die Beschäftigung mit der Thematik erleichtern kann. Sehr illustrativ sind zehn Fallgeschichten, die in eindrucksvoller Form die Symptomatik und den Ver-lauf der Störungen bei betroffenen Patienten darstellen.

Ford, CM.: The Somatizing Disorders. New York, Elsevier, 1983

Es handelt sich um ein flüssig geschriebenes und durch viele Beispiele anschaulich aufbereitetes Buch, in dem sozialpsy-chologische und intrapsychische Aspekte der Somatisierung in den Vordergrund gestellt werden. Der Autor geht sehr ausführlich auf normalpsychologische Prozesse der Krank-heitsbewältigung ein und skizziert Modelle, in denen die Bedeutung der Patienten- und Krankenrolle sowie der ver-mutete «Krankheitsgewinn» sowohl durch familiäre und berufliche Konsequenzen als auch durch die Besonderheiten des Arzt-PatientenVerhältnisses betont werden. Ford ver-wendet einen Somatisierungsbegriff, der deutlich über das Konzept der somatoformen Störungen nach DSM-III-R hin-ausgeht. Ein eigenständiges Kapitel ist der Hysterie und den Konversionsreaktionen gewidmet, wobei die Somatisierungs-störung in ihrer modernen Form noch nicht aufgegriffen wird. Weitere Kapitel behandeln Hypochondrie, organische Schmerzsyndrome, artifizielle oder vorgetäuschte Störungen, Simulation, Münchhausen-Syndrom sowie Störungen im Zu-sammenhang mit posttraumatischen Beeinträchtigungen. Kritisch ware anzumerken, daß umstrittene Konzepte, wie das der somatisierten Depression, oder zum Teil auch psy-chodynamische Überlegungen in den sehr breit angelegten diagnostischen und therapeutischen Ansatz integriert sind und oft etwas unvermittelt nebeneinander stehen.

Bass, CM. (Hrsg.): Somatization - Physical Symptoms and Psychological Illness. Oxford, Blackwell, 1990

In diesem Buch werden zwölf Beiträge britischer Autoren zusammengefaßt, die allesamt eine längere klinische Erfah-

Neuigkciten vom Buch- und Zeitschriftenmarkt

67

rung im Zusammenhang sowohl mit somatisierenden Patien-ten als auch mit eigenen Studien zu diesem Themenbereich haben. Es wird bewußt versucht, somatische und psychologi-sche Aspekte herauszuarbeiten, um das Ineinandergreifen von Medizin und Psychologie gerade im Bereich der somato-formen Störungen deutlich zu machen. Das Buch enthält informative Zusammenfassungen zur Klassifikation der somatoformen Störungen, zum diagnostischen und therapeu-tischen Vorgehen und zur Relevanz der Somatisierung im all-gemeinärztlichen Bereich. In weiteren Beiträgen wird auf spezielle Somatisierungsformen eingegangen: chronische Erschöpftheit, abdominale Schmerzen, kardiorespiratorische Syndrome, Konversionssymptome sowie gynäkologische und dermatologische Beschwerden. Damit verläßt das Buch den heute in der Diagnostik favorisierten Ansatz eines globalen Somatisierungssyndroms und grenzt nach Organsystemen unterschiedene Störungen voneinander ab. Die einzelnen Kapitel überschneiden 
sich zum Teil erheblich, etwa bezüg-lich prädisponierender Faktoren, diagnostischer Methoden oder therapeutischer Vorgehensweisen, so daß zwar jeder Beitrag für sich lesenswert ist, oftmals jedoch ein «roter Faden» zur Verbindung der Einzelbeiträge wünschenswert ware.

Creed, E; Mayou, R.; Hopkins, A. (Hrsg.): Medical Symptoms Not Explained by Organic Disease.

London, Royal College of Psychiatrists and Royal College of Physicians of London, 1992 Dieses Buch ist in erster Linie das Ergebnis einer Konferenz, die vom Ärzteverband und vom Psychiaterverband in Groß-britannien durchgeführt wurde. Es zielt auf eine verbesserte Koordination somatischer, psychiatrischer und psychothera-peutischer Ansätze. Es gliedert sich in verschiedene Kapitel zu abdominalen Beschwerden, Brustschmerzen mit Palpita-tionen und chronischen Kopfschmerzen. Daneben werden ein Modell für funktionelle Störungen dargestellt sowie eini-ge therapeutische Möglichkeiten kurz skizziert (der Ansatz der Reattribution, der kognitiv-verhaltenstherapeutische Ansatz, der Einsatz von Antidepressiva). Insgesamt ist das Buch in weiten Teilen nach den betroffenen Organsystemen gegliedert, wobei in der Regel in Nebensätzen darauf hinge-wiesen wird, daß die Patienten häufig auch über Beschwerden in anderen Organsystemen klagen. In diesem Buch werden somit weniger die grundsätzlichen Neuerungen in der Diagnostik nach DSM-III bzw. DSM-III-R berücksichtigt, so daß zum Teil auch Überlappungen zu anderen Bereichen, wie Panikstörungen, Kopfschmerzen und Colon irritabile, vorkommen, die in der ursprünglichen Definition somatoformer Störungen nicht enthalten sind. Der große Vorteil dieses kleinen Büchleins liegt darin, daß der Leser sehr schnell einen präzisen Überblick über einzelne Aspekte erhält, teilweise Einblick in aktuell laufende For-schungsbemühungen erfährt und durch die Literaturhinweise Anhaltspunkte zur Vertiefung der Thematik erhält.

Rief, W; Hiller, W: Somatoforme Störungen. Bern. Huber. 1992

Hierbei handelt es sich um das bislang einzige deutschspra-chige Werk zu somatoformen Störungen. Es gliedert sich in einen Einleitungsteil, in dem in Kurzfassung die nachfolgend im Detail dargestellten Informationen präsentiert werden. Danach folgt ein ausführlicher diagnostischer Teil, der die historische Entwicklung, den aktuellen Stand, Komorbidität und psychometrische Aspekte darstellt. Auch die Neuerungen durch ICD-10 werden berücksichtigt. Es wird deutlich, daß die große Bedeutung somatoformer Störungen vor allem im gesundheitspolitischen Bereich liegt. Ein erheblicher Teil der Arztbesuche geht auf vorübergehende oder chronifizier-te körperliche Beschwerden zurück, die nicht auf eine organische Ursache zurückzuführen sind. Die Autoren stellen in einem weiteren Gliederungspunkt die verschiedenen Ein-flußvariablen im Sinne von prädisponierenden Faktoren, aus-lösenden Bedingungen und aufrechterhaltenden Variablen dar. Danach werden verschiedene psychologische und psy-chopharmakologische Ansätze beschrieben. Da es sich um eine Monographie handelt, konnten die einzelnen Kapitel konsequent aufeinander aufgebaut werden. Es wurde des weiteren viel Mühe darauf verwendet, das Buch optisch ansprechend zu gestalten und inhaltlich aufzulockern, um zahlreiche interessante Befunde gut lesbar darzustellen. Pennebaker, J.W.: The Psychology of Physical Symptoms. Berlin, Springer, 1982 Pennebaker ist im engen Sinne kein Kliniker, und dieses Buch ist nicht unmittelbar am Konzept der somatoformen Störungen orientiert. Trotzdem nehmen wir es in die Litera-turübersicht mit auf, da es zahlreiche Informationen zur Psychologie der Symptomwahrnehmung enthält, die in anderen Arbeiten zu kurz kommen. Es fasziniert dadurch, daß ein gänzlich anderer Zugang zur Thematik gewählt wird, nämlich der der Wahrnehmungs- bzw. Kognitionspsychologie. Der Kliniker findet zahlreiche Anhaltspunkte, die die Grund-lagenpsychologie für das Verständnis 
klinischer Symptoma-tiken bietet. So begründet Pennebaker durch mehrere Expe-rimente, wie bei fehlender externaler Stimulation internale, körpereigene Stimuli verstärkt wahrgenommen werden. Dies könnte bedeuten, daß psychosoziale Isolation bzw. Unter-stimulation ein Risikofaktor für die Entwicklung somatoformer Störungen darstellt. Familienkonflikte, soziale Unsicher-heit oder Symptomverstärkung werden in ihrer engen Ver-flechtung mit psychophysiologischen Parametern dargestellt, und entsprechende Experimente werden aufgeführt. Somit ist dieses Buch für alle geeignet, die mehr über psychologische Bedingungen von körperlichen Beschwerden erfahren wollen.

68

Neuigkeiten vom Buch- und Zeitschriftenmarkt

Einzelne Artikel

Escobar, J.I.; Rubio-Stipec, M.; Canino, G.; Karno, M.: Somatic Symptom Index (SSI): A New and Abriged Somatization Construct. J Nerv Ment Dis 1989;177:140-146

Einen bedeutenden Stellenwert im Zusammenhang mit Untersuchungen zur klinischen Relevanz des Somatisie-rungssyndroms kommt den Arbeiten von Javier I. Escobar und Mitarbeitern zu, die sich zu Beginn der 80er Jahre inten-siv mit transkulturellen Unterschieden zwischen Weißen und Lateinamerikanern beschäftigten und später wieder führend an der Auswertung von Daten zu somatoformen Störungen im Rahmen der großen amerikanischen «Epidemiologic Catchment Area»-Studie beteiligt waren. Von Escobar stammt das vor allem für den klinischen Bereich interessante Konzept des «Somatic Symptom Index» (SSI), das eine enor-me Ausweitung der sehr restriktiven Definition der Somati-sierungsstörung im DSM-III-R vorsieht. Nach dem SSI liegt eine klinisch relevante Somatisierung nicht erst ab einer Mindestzahl von 13 somatoformen Symptomen vor (wie bei der Somatisierungsstörung), sondern bereits bei vier (bei Männern) bzw. sechs (bei Frauen) Symptomen. In der hier aufgeführten Arbeit führen Escobar und Mitarbeiter den Nachweis, daß Personen mit SSI ähnliche klinische Merk-male etwa bezüglich Lebensbeeinträchtigung, Risikofaktoren und Prognose aufweisen wie Patienten mit dem kompletten Bild der Somatisierungsstörung. Das SSI-Konzept ist bereits in den letzten Jahren in der Literatur aufgegriffen worden, und es ist zu erwarten, daß es auch in Zukunft Studien und klinische Fragestellungen starker beeinflussen wird als das heutige Konzept der

Somatisierungsstörung.

Sharpe, M.; Bass, C: Pathophysiological Mechanisms in Somatization.

Int Rev Psychiatry 1992;4:81/797

Die diagnostischen Kriterien nach DSM-III-R schließen diejenigen körperlichen Symptome bei somatoformen Störungen aus, die auf einem nachweisbaren pathophysiolo-gischen Mechanismus oder auf einer Gewebeschädigung be-ruhen (zum Beispiel Spannungskopfschmerz, Colon irritabile). Diese Ausschlußkriterien können mißverständlich sein, da sie vermuten lassen, es gäbe auch körperliche Symptome ohne organische Korrelate. Wahrscheinlicher ist jedoch, daß auch bei funktionellen körperlichen Beschwerden zwar nicht pathologische, jedoch nachweisbare physiologische Verände-rungen auftreten. Dies kann eine erhöhte Muskelspannung sein, veränderte Durchblutung, veränderte Atmung, neuro-logische oder hormonelle Veränderungen und vieles mehr. Diese und weitere physiologische Mechanismen, die bei Somatisierungsprozessen beteiligt sein können, werden in der Arbeit von Sharpe und Bass ausführlich dargestellt. Der Le-ser erhält deshalb viele Informationen, die vor allem dann von Bedeutung werden, wenn man Patienten psychophysio-logischer Störungsmodelle vermitteln 
möchte. Auch wird be-tont, daß die Symptome «real» sind und daß Annahmen wie «die Symptome sind eingebildet» wenig hilfreich und diskri-

minierend sind. Die Arbeit wird durch ein abschließendes Kapitel mit Implikationen für die Behandlung, für den Ge-sundheitsdienst, für die Weiterbildung und für die Weiterent-wicklung von Klassifikationssystemen in einen größeren Rahmen gestellt.

Sharpe, M.; Peveler, R.; Mayou, R.: The Psychological Treatment of Patients with Functional Somatic Symptoms: A Practical Guide. J Psychosom Res 1992;36:515-529

Aufbauend auf dem kognitiv-verhaltenstherapeutischen Störungsmodell vermittelt diese Arbeit praktische Hinweise für die psychologische Behandlung von Personen mit körperlichen Symptomen ohne organische Erkrankung. Dies be-ginnt mit Ratschlägen an Ärzte, wie der negative Untersu-chungsbefund dem Patienten am besten zu vermitteln ist und wie eine Überweisung an den Psychologen oder Psychothera-peuten sinnvoll erfolgen kann. Dieser wird in der Regel mit einer ausführlichen Diagnostik von Symptomen, Kognitio-nen, Emotionen, Verhaltenskomponenten und möglichen al-ternativen psychophysiologischen

Erklärungsmodellen den Behandlungsprozeß fortsetzen. Edukative Maßnahmen, ge-leitetes Entdecken, Verhaltensexperimente und provozierte Erfahrungen sollen schließlich zu einer Umbewertung von katastrophisierenden Gedanken zu körperlichen Symptomen sowie zu einer Defokussierung der Aufmerksamkeit weg von Körperprozessen führen. Unterstützt wird dies durch Sym-ptomtagebücher. Somit findet der Leser eine prägnante und anschauliche Darstellung des therapeutischen Prozesses.

Salkovskis, P. M.: Somatic Problems; in Hawton, K.; Salkovs-kis, P.M.; Kirk, J.; Clark, DM. (Hrsg.): Cognitive Behaviour Therapy for Psychiatric Problems. A Practical Guide. Oxford, Oxford Medical Publications, 1989

Einen ausführlichen Artikel über das kognitiv-verhaltens-therapeutische Vorgehen findet der Leser in dieser Arbeit. Im Gegensatz zum vorgenannten Artikel liegt der Schwerpunkt weniger in der systematisierten Darstellung des therapeutischen Ablaufs als vielmehr in der anschaulichen und durch zahlreiche Fallbeispiele erläuterten Darstellung der klinischen Praxis. Es werden zahlreiche sinnvolle Vorgehens-weisen und Formulierungen im Umgang mit Patienten mit somatischen Krankheitsmodellen explizit aufgeführt, so daß die einzelnen Interventionen durch den Wortlaut von Aus-schnitten aus Therapiesitzungen deutlich werden. Somit kann diese Arbeit nach Ansicht der Referenten als die anschau-lichste der uns momentan bekannten Arbeiten zur Psycho-therapie bezeichnet werden. Obwohl der Schwerpunkt des Artikels eher im Bereich Hypochondrie liegt, wird auch auf Besonderheiten und Kognitionen von Personen mit Somati-sierungsstörungen, körperdysmorphen Störungen, Schmer-zen oder klassisch-psychosomatischen Erkrankungen, wie Asthma bronchiale, eingegangen.

Neuigkeiten vom Buch- und Zeitschriftenmarkt

69

Zusammenfassung

Bei der Durchsicht der Literatur wird deutlich, daß man im Bereich somatoformer Störungen noch weit von dem Kennt-nisstand anderer psychischer Störungen (wie Panikstörungen oder Depressionen) entfernt ist. Trotzdem lassen sich bereits zahlreiche Befunde herausgreifen, die darauf hindeuten, daß die klassifikatorische Neufassung durch das Konzept «soma-toforme Störungen» einen sinnvollen neuen Weg beschreitet. Das komplette Bild einer Somatisierungsstörung stellt ein stabiles, zur Chronifizierung neigendes Syndrom dar, was nach dem bisherigen Kenntnisstand nur in Ausnahmefällen durch bekannte somatische Erkrankungen 
erklärt werden kann. Somatoforme Symptome treten oftmals über mehrere Organsysteme verteilt auf. Hierarchische Ansätze, wie z.B. das der «somatisierten Depression», sollten vermieden werden. Vieles spricht bei der Entwicklung zukünftiger Klas-sifikationssysteme dafür, zwischen der Gruppe der Somatisierungsstörung und der Gruppe der undifferenzierten somatoformen Störung eine Gruppe von multiplen somatoformen Symptomen einzuführen, wie sie zum Beispiel in den Arbei-ten von Escobar vorgeschlagen wird. Bei der Entstehung und Aufrechterhaltung somatoformer Störungen lassen sich bereits einige Risikovariablen identifizieren, z.B. frühe Krank-heitsmodelle, sexuelle Traumata, familiäre Belastungen durch Alkoholismus und Soziopathie, kritische Lebensereig-nisse und reduzierte Bewältigungsstrategien für Belastungen. Häufig gehen somatoforme Störungen mit Depressionen einher, jedoch auch mit Angststörungen und anderen psychi-schen Störungen. Der kognitiv-verhaltenstherapeutische Ansatz kann bereits einige erfolgreiche Hilfen anbieten, muß jedoch noch intensiv weiterentwickelt werden.

27. Verhaltenstherapiewoche, Frühjahrsprogramm

\section{7.-11.5.1993 in Kiel}

Thema der Eröffnungsveranstaltung: Yerhaltenstherapie in der Psychiatrie

28. Verhaltenstherapiewoche, Sommerprogramm

25.-29.6.1993 in Dresden

Thema der Eröffnungsveranstaltung: Verhaltensmedizin - eine neue Perspektive in der Behandlung körperlicher Krankheit

Es werden Kurse und Serainare zu folgenden Themenbereichen angeboten:

Diagnostische und therapeutische Verfahren

Kurse für Pflegepersonal, Arbeits- und Beschäftigungstherapeuten

Kurse zum Thema der Eröffnungsveranstaltung

Störungen und Therapie bei Kindern und Jugendlichen

Ausgewählte Krankheits- und Störungsbilder und deren Therapie

Verhaltensmedizin und Rehabilitation

Fortbildungszyklus für Leiter von Kursen zur Prävention von Herz- und Kreislauferkrankungen

Auskunft und Anmeldung:

IFT Institut für Therapieforschung

Verhaltenstherapiewoche

Parzivalstraße 25

D-8000 München 40

Tel: 089/360804-22

Fax: 089/360804-69 